\title{
Vulnerability Analysis and Consequences of False Data Injection Attack on Power System State Estimation
}

\author{
Jingwen Liang, Student Member, IEEE, Lalitha Sankar, Member, IEEE, and Oliver Kosut, Member, IEEE
}

\begin{abstract}
An unobservable false data injection (FDI) attack on AC state estimation (SE) is introduced and its consequences on the physical system are studied. With a focus on understanding the physical consequences of FDI attacks, a bi-level optimization problem is introduced whose objective is to maximize the physical line flows subsequent to an FDI attack on DC SE. The maximization is subject to constraints on both attacker resources (size of attack) and attack detection (limiting load shifts) as well as those required by DC optimal power flow (OPF) following SE. The resulting attacks are tested on a more realistic nonlinear system model using AC state estimation and ACOPF, and it is shown that, with an appropriately chosen sub-network, the attacker can overload transmission lines with moderate shifts of load.
\end{abstract}

Index Terms - false data injection, state estimation, optimization, vulnerability analysis.

\section{INTRODUCTION}

W ITH the increasing integration of real-time monitoring, sensing, control, and communication, the electric power systems are becoming increasingly efficient and controllable. However, the tight integration also makes the system more vulnerable to cyber attacks with potentially serious physical consequences. Therefore, assessment and evaluation of possible attacks and consequences before an actual attack happens is extremely instructive to the utilities: procedures for detecting potential attack incidents is an important supplements to the secure operation of the power system.

There is much interest in studying cyber attacks on the electric power system. This includes attacks on system states [1]-[6], system topology [7], [8], generator dynamics [9], and energy markets [10]-[12]. While several classes of cyberattacks have been identified, consequences of such attacks on the electric power system are less understood. It is this aspect that we focus on in this paper. To this end, we introduce a class of false data injection (FDI) attacks on AC state estimation (SE) designed to cause physical line overflows. In fact, such an attack can potentially lead to cascading failures since a sustained attack can ensure that the physical line overflow is not detected through the cyber measurements.

\section{A. Contributions}

The contributions of this paper are two-fold. First, we introduce a sophisticated unobservable attack on AC state

J. Liang, L. Sankar, and O. Kosut are with the Department of Electrical, Computer and Energy Engineering, Arizona State University, Tempe, AZ 85281 USA, e-mail: jliang19@asu.edu. Isankar@asu.edu and okosut@asu.edu This work is supported in part by NSF grant CPS-1449080. estimation that takes into account the sequential data processing functionalities in the cyber layer of the electric power system (see Fig. 11). Our attack models a sophisticated attacker with access to measurements in a small sub-network and with the intention of creating significant changes to the physical network that can have potentially damaging consequences, if undetected. Enabling physical consequences requires the attacker to change measurements that leads to redispatch, and eventually, line overloads. To this end, we formulate a bi-level attack optimization problem with the objective of causing a physical line overflow via an unobservable attack on AC state estimation subject to constraints on: (i) number of meters to attack (limited resources constraint) and (ii) load shifts (to limit operator detection). Since a line overflow requires modeling the system level redispatch subsequent to the attack, our optimization problem has embedded in it a second level redispatch optimization problem. The second contribution of our work is to highlight the consequences of our proposed attack on a non-linear system model with AC SE and ACOPF. We use the optimal attack vector obtained from our optimization problem to do so. We show that our attack model can successfully lead to line overflows for an RTS-24bus system with moderate load shifts and attack sizes.

\section{B. State of the Art}

FDI attacks have gained much interest in the literature starting from Liu et al.'s work on unobservable attacks on DC SE [1]. Their work shows that an attacker can change the system state without being detected by the bad data detection algorithm within SE if the attack vector is chosen judiciously to mimic typical SCADA measurements. Kosut et $a l$. discuss the trade-off between maximizing estimation error at the control center and minimizing detection probability of the attack [3].

For attacks restricted to a sub-network of the system network graph, the authors in [13] introduce an algorithm to determine an attack subgraph and show that such a subnetwork must be bounded by buses with injections. Furthermore, the authors also show that a sophisticated attack using AC SE requires the attacker to estimate the system states for its subgraph. Recently, in [14], we build upon [13] to introduce an AC attack restricted to a subgraph and show that it suffice for the attacker to perform local SE to launch an unobservable attack.

In this paper we extend [14] to study attack consequence. To this end, we use an attack subgraph and determine the optimal 
attack via a bi-level optimization problem. Bi-level attack optimization problems in the context of attacks are considered in [15]-[17]. In all cases, the optimization problems include both the attacker's goal (unobservable attack on DC SE) as well as the ensuing system response (OPF), leading to a bi-level optimization problem. However, the goal of the optimization in the aforementioned papers is to increase the operating costs for the system. While costs are relevant to the electric power system operation, cyber attacks with physical consequences can be more damaging.

The optimization problems in [16] and [17] take into account the fact that FDI attacks lead to an inevitable load shift at the buses in the subgraph and include a constraint on the load shift magnitude to limit detection. In this paper, we take this a step further and restrict not only the load shift magnitude but also the size of attack subgraph to simultaneously model the observability and limited resources constraints.

The remainder of this paper is organized as follows. Sec. II introduces the general system and attack model. Sec. III discusses the different attack strategies for unobservable attacks. Sec. IV presents a bi-level optimization formulation to identify the worst-case overflow attack. Sec. V presents and analyzes the numerical results for a test system. Sec. VI draws the conclusion of this paper and presents the direction of future works.

\section{PROBlem Formulation: SySTEM AND ATTACK MODEL}

\section{A. Temporal nature processing of the grid}

Fig. 1 illustrates the temporal nature of processing in the grid and the attack model. Assume a system with $n_{b}$ buses, $n_{b r}$ branches, and $n_{g}$ generators. Active and reactive load of each buses are represented by $P_{L}$ and $Q_{L}$, respectively. Measurement and estimated measurement residue are denoted as $z$ and $r$, respectively. In the bad data detector, $\tau$ is the residue threshold and $x=[V, \theta]^{T}$ is the system state, where $V$ is bus voltage magnitude and $\theta$ is bus voltage angle. The function $h(\cdot)$ denotes the non-linear function that gives the measurements. This function depends only on the system topology. Estimated values are denoted with a hat, e.g. $\hat{x}, \hat{V}, \hat{\theta}$.

As shown in Fig. 1, generation dispatch control decisions made at the control center depend on the noisy measurements provided by the SCADA system. If these measurements are corrupted by an attacker and pass the bad data detector, they can directly influence the control decisions for the next time interval. Since the process occurs in the same manner for each time $t$, we drop the functional dependence on $t$ for the rest of this section. The major blocks shown in Fig. 1 are discussed in detailed in the following subsections.

\section{B. Measurements}

The AC measurement model follows the non-linear relationship

$$
z=h(x)+e
$$

where $z, e$ and $x$ are $m \times 1, m \times 1$ and $n \times 1$ vectors with entries $z_{i}, e_{i}$ and $x_{k}$, respectively $i \in\{1, \ldots, m\}$ and $k \in$
$\{1, \ldots, n\} . z_{i}$ is the $i^{\text {th }}$ measurement of the system: line power flows, bus voltage and line current magnitude, etc. $e_{i}$ is the $i^{\text {th }}$ measurement error, assuming to be independent and Gaussian distributed with 0 mean and $\sigma_{i}^{2}$ covariance.

\section{State estimation}

As illustrated in Fig. 1, all raw measurements are first passed through an observability check. If there are enough measurements, the system will be observable; otherwise, the system is divided into several observable islands.

State estimation is used to determine the most likely state of the system given the available noisy measurements. In AC state estimation, the state variables are solved as a least square problem with an objective function [18]

$$
\hat{x}=\arg \min J(x)=(h(x)-z)^{T} R^{-1}(h(x)-z)
$$

where $R=\operatorname{diag}\left\{\sigma_{1}^{2}, \sigma_{2}^{2}, \ldots, \sigma_{m}^{2}\right\}$ and $\hat{x}=[\hat{V}, \hat{\theta}]^{T}$ is the estimated state.

Subsequent to SE, the bad data detector filters noisy measurement and guarantees the accuracy of estimation, using $\chi^{2}$ test.

\section{AC and DC optimal power flow}

The AC optimal power flow (OPF) takes the following form:

$$
\begin{array}{cl}
\underset{x}{\operatorname{minimize}} & f\left(P_{G}\right) \\
\text { subject to } & G(x)=0 \\
& F(x) \leq 0 \\
& x_{\max } \leq x \leq x_{\max }
\end{array}
$$

where $f($.$) is the generation cost function and x=$ $\left[V, \theta, P_{G}, Q_{G}\right]^{T}$ is the variable of the optimization problem. Inequality constraint, i.e. the line thermal limits, is denoted as $F$ and equality constraint, i.e. the node power balance is denoted as $G$. Both $F$ and $G$ are non-linear constraints, since there are active and reactive power involved.

DCOPF approximates $G$ and $F$ around $V=1, \theta=0$ by their first order Taylor expansion:

$$
\begin{array}{cl}
\underset{\theta, P_{G}}{\operatorname{minimize}} & f\left(P_{G}\right) \\
\text { subject to } & -H_{1} \theta+P_{G}-P_{L}=0 \\
& -P^{\max } \leq H_{2} \theta \leq P^{\max } \\
& P_{G}^{\min } \leq P_{G} \leq P_{G}^{\max } .
\end{array}
$$

where

- $H_{1}$ is the matrix of dependencies between power injection and state $\theta$.

- $H_{2}$ is the matrix of dependencies between branch power flow and state $\theta$.

- $P^{\max }$ is the thermal limit.

- $P_{G}^{\min }$ and $P_{G}^{\max }$ are the generator capacity lower and upper limit, respectively. 


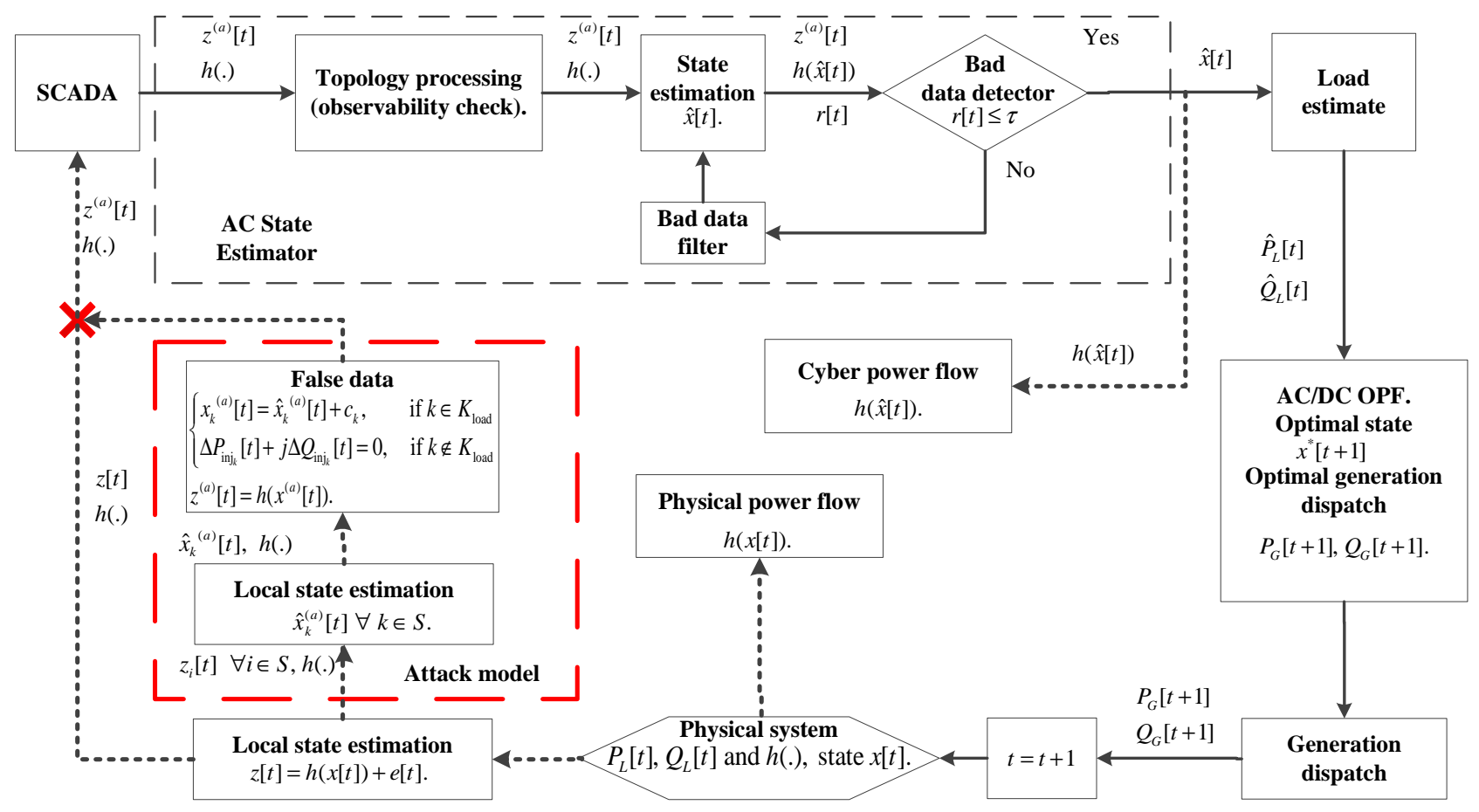

Figure 1. Illustration of temporal processing of the grid and attack model.

\section{E. Attack model}

We first assume that the attacker has following capabilities:

1) The attacker has access to all measurements and topology information of a small area $\mathcal{S}$ bounded by buses. The set of all measurement indices in $\mathcal{S}$ is denoted as $\mathcal{I}_{\mathcal{S}}$ and the set of all state indices in $\mathcal{S}$ is denoted as $\mathcal{K}_{\mathcal{S}}$.

2) The attacker can change or replace all measurements in $\mathcal{S}$.

3) The attacker has computational capability.

As discussed in [14], according to (1), suppose the $i^{\text {th }}$ measurement prior to attack is $z_{i}=h_{i}(x)+e_{i}$, the general attack model changes the $i^{\text {th }}$ measurement $z_{i}$ to $z_{i}^{(a)}$ such that

$$
z_{i}^{(a)}= \begin{cases}z_{i} & \text { if } i \notin \mathcal{I}_{\mathcal{S}} \\ \tilde{z}_{i} & \text { if } i \in \mathcal{I}_{\mathcal{S}}\end{cases}
$$

where $\tilde{z}_{i}$ is chosen by attacker.

\section{ATTACK STRATEGY}

\section{A. Unobservable attack}

Definition 1. An attack is unobservable for a measurement model $h(\cdot)$ if, in the absence of measurement noise, there exists a $c \neq 0$ such that $z_{i}^{(a)}=h_{i}(x+c)$ for all $i$.

Therefore, for the attacker to execute an unobservable attack, again assuming no measurement noise, (9) becomes

$$
z_{i}^{(a)}= \begin{cases}z_{i} & \text { if } i \notin \mathcal{I}_{\mathcal{S}} \\ h_{i}(x+c) & \text { if } i \in \mathcal{I}_{\mathcal{S}} .\end{cases}
$$

From (10), if the $k^{\text {th }}$ state $x_{k}$ is required to compute $h_{i}(x)$ for any $i \notin \mathcal{I}_{\mathcal{S}}$, then for any unobservable attack the corresponding $k^{\text {th }}$ entry in attack vector must satisfy $c_{k}=0$.
That is, for an attack region $\mathcal{S}$, not all the bus states in it can be changed. The attack region must be bounded by a set of buses without state changes however with measurement changes. To identify such a collection of one or more buses in $\mathcal{S}$, we first distinguish between two types of buses based on the presence of load. We henceforth identify buses with load as load buses. $\mathcal{K}_{\text {load }}$ denotes the bus indices of load bus. An attacker can attack either type of bus. However, since the injections of non-load buses are known to the control center, attacking a non-load bus implies that the measurements at the closest load buses also need to be changed to ensure that the nodal power balance is maintained. In [13], a method is introduced to identify a subgraph of the network that allows an attacker to perform an unobservable attack. We use a similar method, as summarized as follow. Let $k$ be a target load bus, the corresponding single-target-bus attack subgraph $\mathcal{S}_{k}$ is constructed by following steps:

1) Include bus $k$ in $\mathcal{S}_{k}$.

2) Extend $\mathcal{S}_{k}$ from bus $k$ by including all buses and branches that are connected to bus $k$.

3) If there is a non-load bus on the boundary of $\mathcal{S}_{k}$, extend $\mathcal{S}_{k}$ to include all adjacent buses of such a boundary bus.

4) Repeat (3) until all buses on the boundary are load buses or $\mathcal{S}_{k}$ can not be extended anymore.

The steps above give an attack subgraph that includes the target load bus and is bounded by load buses. Fig. 2 shows two simple examples of single-target-bus attack subgraphs. The choice of the final attack subgraph $\mathcal{S}$, however, can be a union of several single-target-bus attack subgraphs:

$$
\mathcal{S}=\bigcup_{k: c_{k} \neq 0 \cap k \in \mathcal{K}_{\text {load }}} \mathcal{S}_{k} .
$$

This choice of attack subgraph results in estimated load 


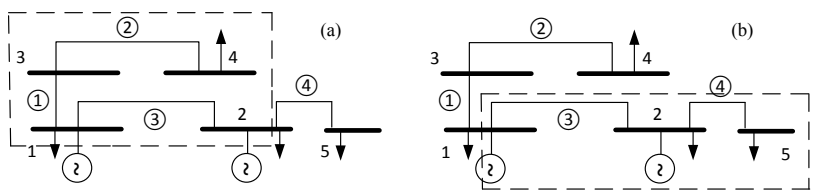

Figure 2. Examples of single-target-bus attack subgraph. Fig. 2a shows the subgraph with target bus 1 and Fig. 2b shows the subgraph with target bus 2 .

changes at all load bus within $\mathcal{S}$ while no net load changes in the system.

\section{B. DC attack}

Since 10 is nonlinear and generally hard to solve, it is reasonable for the attacker to first consider a simplified DC attack. As [1] demonstrated, by knowing system Jacobian matrix $H$, an attacker can intelligently construct an unobservable attack vector $a=H c$ such that $z_{i}^{(a)}=z_{i}+a$.

Thus, (10) becomes

$$
z_{i}^{(a)}= \begin{cases}z_{i} & \text { if } i \notin \mathcal{I}_{\mathcal{S} P} \\ z_{i}+H_{(i,:)} c & \text { if } i \in \mathcal{I}_{\mathcal{S} P}\end{cases}
$$

where, $\mathcal{I}_{P}$ denotes the set of indices of active power measurements, $\mathcal{I}_{\mathcal{S} P}=\mathcal{I}_{\mathcal{S}} \cap \mathcal{I}_{P}$, and $H_{(i,:)}$ denotes the $i^{\text {th }}$ row of $H$.

Though DC attack is easy to construct, it is not an unobservable attack for $\mathrm{AC}$ state estimator. Without taking reactive power flow into account, a DC attack will be detected when $c$ is too large.

\section{AC attack}

From (10), in contrary to DC attack, it seems that the attacker must know all the state values that appear in $h_{i}($.$) , for$ all $i \in I_{\mathcal{S}}$, to construct $z_{i}$ precisely. However, this information is not available to the attacker. Thus, attacker can use the following steps to construct $z_{i}^{(a)}$ :

1) The attacker first chooses the non-zero entries in $c$ only for the load buses. These non-zero entries correspond to the center buses for the attack subgraph.

2) Use the protocol in Sec. III-A and choose $\mathcal{S}$ for the desired attack.

3) Given the measurements that are available to the attacker in $\mathcal{S}$, perform local AC state estimation to find $\hat{x}_{k}^{(a)}$. The slack bus may be chosen arbitrarily among all load buses.

4) For all load buses $k$, set $x_{k}^{(a)}=\hat{x}_{k}^{(a)}+c_{k}$.

5) Since the injection of non-load buses can not be changed, the states of non-load buses are dependent on the state of all the buses that connected to them. Therefore, the attacker has the nodal balance equation for each non-load bus $k$ in $\mathcal{S}$ :

$$
\begin{aligned}
& P_{\mathrm{inj}_{k}}=V_{k} \sum_{i \in \mathcal{N}_{k}} V_{i}\left(G_{k i} \cos \theta_{k i}+B_{k i} \sin \theta_{k i}\right) \\
& Q_{\mathrm{inj}_{k}}=V_{k} \sum_{i \in \mathcal{N}_{k}} V_{i}\left(G_{k i} \sin \theta_{k i}-B_{k i} \cos \theta_{k i}\right)
\end{aligned}
$$

where $G_{k i}+j B_{k i}$ is the $(k, i)^{\text {th }}$ entry of the complex bus admittance matrix and $\theta_{k i}=\theta_{k}-\theta_{i}$ is the angle difference between bus $k$ and $i$. These equations can be solved by iterative methods such as Newton-Raphson method.

6) With all the computed state information, the attacker can therefore compute the false measurements $z^{(a)}$ such that

$$
z_{i}^{(a)}= \begin{cases}z_{i} & \text { if } i \notin \mathcal{I}_{\mathcal{S}} \\ h_{i}\left(x^{(a)}\right) & \text { if } i \in \mathcal{I}_{\mathcal{S}} .\end{cases}
$$

\section{OPTIMIZATION PROBLEM FOR THE WORST-CASE LINE OVERLOAD ATTACK}

The aim of the unobservable attack is to maximize the physical line flow for a chosen line in the attack subgraph. However, the attacker, in general, has limited resources to change states; furthermore, the attacker would also like to design the attack to avoid detection over the various computing units in EMS. This leads to a constrained optimization problem. Specifically, we model the two conflicting goals of the attacker as follows: the limited resource constraint is modeled by a sparsity constraint in which we limit the number of center buses at which states can be changed. The detectability constraint is modeled by limiting the cyber load shifts that result from the FDI attacks. This is because a large deviation in estimated load from normal operational values will be detected as an anomalous event by the operators. The sparsity constraint capturing the limited resource is modeled as an $l_{0}$-norm constraint. This is, in general, intractable, and therefore, we relax it to an $l_{1}$-norm constraint. In addition to the two constraints, since the physical line flow is a consequence of the control center re-dispatch generation, the attack optimization process has to include the OPF subsequent to state estimation as a sub-problem. The resulting problem is a bi-level optimization problem.

Generally, an optimal dispatch can be the result of different load patterns. As a result, there are numerous solutions of attack vector that lead to the same physical line flow on the target line. Among these, the goal of the optimization is to choose the one with the smallest $l_{1}$-norm, and hence, $l_{0}$ norm to satisfy the limited resources constraint. This, in turn, requires a second entry in the objective function where we determine the sparest attack vector among the same maximal power flow on the target branch.

The attacker's influences over the system can be formulated as an optimization problem (with attacker's objective) embedded with a sub-problem (with operator's objective). Similar to the authors in [15], [16], we model the optimal attack problem as a bi-level optimization problem with an objective to maximize the power flow on branch $l$ while to change as few states as possible:

maximize $\quad P_{l}-\gamma\|c\|_{0}$

subject to

$$
\begin{aligned}
& P=H_{2}\left(\theta^{\star}-c\right) \\
& -L_{S} P \leq H_{1} c \leq L_{S} P_{L} \\
& \|c\|_{0} \leq N_{0}
\end{aligned}
$$




$$
\begin{aligned}
& \left\{\theta^{\star}, P_{G}^{\star}, R^{\star}\right\}=\arg \left\{\min _{\theta, P_{G}, R} \sum_{g=1}^{n_{g}} f_{g}\left(P_{G_{g}}\right)+\sum_{l=1}^{n_{b r}} \mathcal{P}_{l}\left(R_{l}\right)\right\} \\
& P_{G}-H_{1}(\theta-c)-P_{L}=0 \quad(v) \\
& -P^{\max }-R \leq H_{2} \theta \leq P^{\max }+R \\
& P_{G}^{\min } \leq P_{G} \leq P_{G}^{\max }\left(\lambda^{+}, \lambda^{-}\right) \\
& 0 \leq R
\end{aligned}
$$

where the variables:

$P \quad$ is the $n_{b r} \times 1$ vector of branch power flow;

$c \quad$ is the $n_{b} \times 1$ attack vector;

$\theta, \theta^{\star} \quad$ are $n_{b} \times 1$ state variable vectors and optimal variable solved by DCOPF, respectively;

$P_{G}, P_{G}^{\star} \quad$ are $n_{g} \times 1$ vectors of generation dispatch variable and optimal generation dispatch solved by DCOPF, respectively;

$R, R^{\star} \quad$ are $n_{b r} \times 1$ vectors of the line relaxation variable, and optimal line relaxation solved by DCOPF, respectively;

$v \quad$ is the $n_{b} \times 1$ dual variable vector for all equal constraints in DCOPF;

$\lambda^{+}, \lambda^{-} \quad$ are $n_{b r} \times 1$ dual variable vectors of the upper and lower bound of thermal limits, respectively;

$\alpha^{+}, \alpha^{-} \quad$ are $n_{g} \times 1$ dual variable vectors of the upper and lower bound of generator capacity, respectively;

and the parameters:

$L_{S} \quad$ is the load shift factor;

$P_{L} \quad$ is the $n_{b} \times 1$ vector of active load at each bus;

$N_{0} \quad$ is the $l_{0}$-norm constraint integer;

$H_{1} \quad$ is the $n_{b} \times n_{b}$ matrix of dependencies between power injection measurements and state variables;

$H_{2} \quad$ is the $n_{n b} \times n_{b}$ matrix of dependencies between power flow measurements and state variables;

$f_{g} \quad$ is the cost function of the $g^{\text {th }}$ generator;

$\mathcal{P}_{l} \quad$ is the penalty function of relaxing the $l^{\text {th }}$ line;

$P^{\max } \quad$ is the $n_{b r} \times 1$ vector of line thermal limit;

$P_{G}^{\min }, P_{G}^{\max }$ are $n_{g} \times 1$ vectors of minimum and maximum generator output, respectively;

$\gamma \quad$ the weight of the norm of attack vector $c$.

We define $l_{0}$-norm as appropriate quantities summed over only the load buses. Thus, the $l_{0}$-norm, $\|c\|_{0}$, of the attack vector $c$ is defined as

$$
\|c\|_{0}=\sum_{k \in \mathcal{K}_{\text {load }}}^{n_{b}} 1\left(c_{k} \neq 0\right) .
$$

Recall the goal of optimization is to maximize $P_{l}$ while finding the sparsest attack among all the possible attack vector. Thus, due to the trade-off between the maximum power flow and the corresponding sparest attack vector, thus the optimization objective is $P_{l}-\gamma\|c\|_{0}$. The weight $\gamma$ is chosen to be a small and positive value such it in general contributes minimal to the objective. Note that (17)-(19) are the attack related constraints. The constraints in (17) model the unobservability of the attack and the constraints in (18)- 19 model the attacker's limited ability: the attacker can alter up to $N_{0}$ states (not necessarily alter all of them) and the resulting change in load shift is limited to $L_{S} P_{L}$. A standard DCOPF with a thermal limit relaxation penalty is modeled by (20)(24). The penalty function in 20 ensures the second level OPF converge thus the first level problem to return a solution.

Since (19) is a modified $l_{0}$-norm constraint, it is a complex non-linear constraint and generally non-convex. In this paper, we relax it to a corresponding $l_{1}$-norm constraint as

$$
\|c\|_{1}=\sum_{k \in \mathcal{K}_{\text {load }}}\left|c_{k}\right| \leq N_{1}
$$

where $N_{1}$ is non-negative. Since (26) is a non-linear constraint and we rewrite it as

$$
-c_{k} \leq s_{k}, \quad c_{k} \leq s_{k}, \quad \sum_{k \in \mathcal{K}_{\text {load }}} s_{k} \leq N_{1} .
$$

where $s$ is a slack variable.

For the embedded OPF problem, the optimal solution can be found at the point which satisfies the KKT optimality condition with zero duality gap since it is a convex optimization problem [19]. We use this fact to further replace the embedded DCOPF problem in 20) with its KKT conditions below, along with (21)-24], as

$$
\begin{gathered}
{\left[\lambda^{+} ; \lambda^{-} ; \alpha^{+} ; \alpha^{-} ; \beta\right] \geq 0} \\
\operatorname{diag}\left(\left[\lambda^{+} ; \lambda^{-}\right]\right)\left(\left[H_{2} ;-H_{2}\right] \theta^{\star}-\left[P^{\max }+R^{\star}\right][I ;-I]\right)=0 \\
\operatorname{diag}\left(\left[\alpha^{+} ; \alpha^{-}\right]\right)\left([I ;-I] P_{G}^{\star}-\left[P_{G}^{\max } ;-P_{G}^{\min }\right]\right)=0 \\
-\operatorname{diag}(\beta) R^{\star}=0 \\
\nabla\left(\sum_{g=1}^{n_{g}} f_{g}\left(P_{G_{g}}^{\star}\right)+\sum_{l=1}^{n_{b r}} \mathcal{P}_{l}\left(R_{l}^{\star}\right)\right) \\
+\left[\lambda^{+} ; \lambda^{-}\right]^{T} \nabla\left(\left[H_{2} ;-H_{2}\right] \theta^{\star}-\left[P^{\max }+R^{\star}\right][I ;-I]\right) \\
+\left[\alpha^{+} ; \alpha^{-}\right]^{T} \nabla\left([I ;-I] P_{G}^{\star}-\left[P_{G}^{\max } ;-P_{G}^{\min }\right]\right) \\
-\beta^{T} \nabla R^{\star}+v^{T} \nabla\left[P_{G}^{\star}-H_{1}\left(\theta^{*}-c\right)-P_{L}\right]=0
\end{gathered}
$$

where 29]-31) are the complementary slackness condition for constraint 222-24) and (32) is the partial gradient optimal condition. Though (29)-(31) are non-linear, they have specially distinctive nature. For instance, the $j^{\text {th }}$ equation in (31) can be separated into two conditions associated with a binary variable $\delta_{\beta_{j}}$

$$
\begin{cases}\beta_{j} \geq 0 \text { and }-R_{j}^{\star}=0, & \text { if } \delta_{\beta_{j}}=0 \\ \beta_{j}=0 \text { and }-R_{j}^{\star}<0, & \text { if } \delta_{\beta_{j}}=1 .\end{cases}
$$

In [20], a procedure is proposed to write [33] as a mixed integer problem given as

$$
\delta_{\beta_{j}}=\{1,0\}, \quad \beta_{j} \leq C \delta_{\beta_{j}}, \quad R_{j}^{\star} \leq C\left(1-\delta_{\beta_{j}}\right) .
$$

If $\delta_{\beta_{j}}=0$, substitute (24) and 28) into (34), we have

$$
\delta_{\beta_{j}}=0, \quad 0 \leq \beta_{j} \leq 0, \quad 0 \leq R_{j}^{\star} \leq C_{j} .
$$

Thus, if $C_{j}$ is large enough to not effect the solution of $R_{j}^{\star}$, (35) is equivalent to the complementary slackness when the $j^{\text {th }}$ constraint in 24) is not an active constraint. Similarly, if $\delta_{\beta_{j}}=1$ and substitute (24) and 28) into [39), we have

$$
\delta_{\beta_{j}}=1, \quad 0 \leq \beta_{j} \leq C_{j}, \quad 0 \leq R_{j}^{\star} \leq 0 .
$$


Again, if $C_{j}$ is large enough to not effect the solution of $\beta_{j}$, (36) is equivalent to the complementary slackness when the $j^{\text {th }}$ constraint in (24) is an active constraint. Therefore, 34) is equivalent to 31 .

Thus, the whole problem becomes the mixed-integer linear program

$$
\begin{aligned}
& \text { maximize } \quad P_{l}-\gamma \sum_{k \in \mathcal{K}_{\text {load }}} s_{k} \\
& \text { subject to } \\
& \text { (17)-(18), 21)-24), 277-28), (32) } \\
& \left\{\begin{aligned}
& \delta_{\lambda}^{ \pm}=\{1,0\} \\
& \lambda^{ \pm} \leq C \delta_{\lambda}^{ \pm} \\
&-H_{2} \theta^{\star}+P^{\max }+R^{\star} \leq C\left(1-\delta_{\lambda}^{+}\right) \\
&+H_{2} \theta^{\star}+P^{\max }+R^{\star} \leq C\left(1-\delta_{\lambda}^{-}\right)
\end{aligned}\right. \\
& \left\{\begin{array}{c}
\delta_{\alpha}^{ \pm}=\{1,0\} \\
\alpha^{ \pm} \leq C \delta_{\alpha}^{ \pm} \\
-P_{G}^{\star}+P_{G}^{\max } \leq C\left(1-\delta_{\alpha}^{+}\right) \\
P_{G}^{\star}-P_{G}^{\min } \leq C\left(1-\delta_{\alpha}^{-}\right)
\end{array}\right. \\
& \left\{\begin{array}{l}
\delta_{\beta}=\{1,0\} \\
\beta \leq C \delta_{\beta} \\
R^{\star} \leq C\left(1-\delta_{\beta}\right)
\end{array}\right.
\end{aligned}
$$

where $\delta_{\lambda}^{ \pm}, \delta_{\alpha}^{ \pm}$and $\delta_{\beta}$ are binary variables and $C$ is a large constant.

\section{Simulation Results}

In this section, we run the optimization problem defined in Sec. IV] on the IEEE RTS-24-bus system to find an optimal attack vector $c$. Subsequently, we use this attack vector $c$ to simulate an AC attack described in Sec. III-C and given by (15) against a non-linear system model involving $\mathrm{AC}$ state estimation and ACOPF. AC power flow, AC state estimation, and ACOPF are implemented with MATPOWER toolbox in MATLAB. For the optimization problem, we use CPLEX as the solver.

\section{A. Solution for the optimization problem}

We highlight results of two scenarios for the RTS-24-bus system: one with original rating and one with reduced rating. The one with original rating represents a system without congestion prior to attack and the one with reduced rating represent a congested system.

Second, we define an attack as feasible if the resulting change in power flow is more than $1 \%$ of the power flow value prior to the attack. This is to distinguish the cases with no or minor changes on target branch power flow $P_{l}$ after attack from those with large changes. We furthermore define a feasible attack to be successful if the target branch is overloaded after attack. We choose $\gamma$ to be $1 \%$ of the original power flow value of the target branch.

Figs. 3 and 4 illustrate relevant statistics for the noncongested and congested systems, respectively, when the $N_{1}$ constraint is set to be infinite. That is, the attacker has control over all measurements of the system and can change as many measurements as it wishes. The congested system is modeled with all branch ratings decreased by $50 \%$. There are three

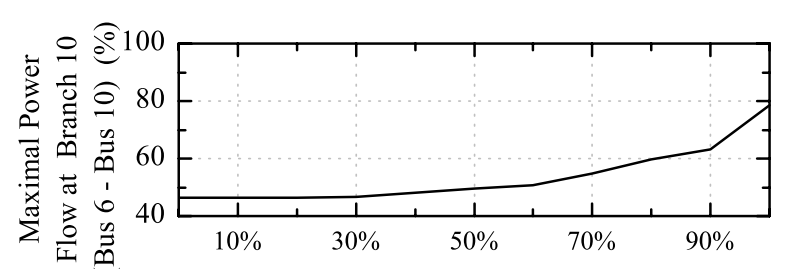

(a)

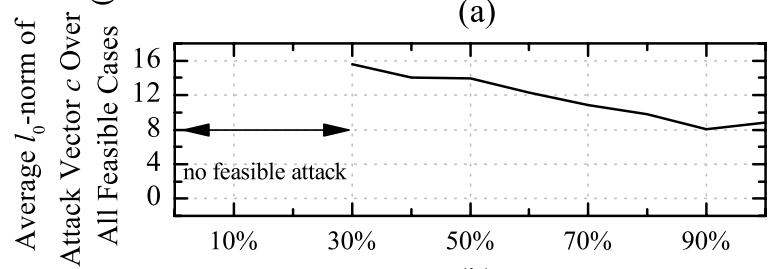

(b)

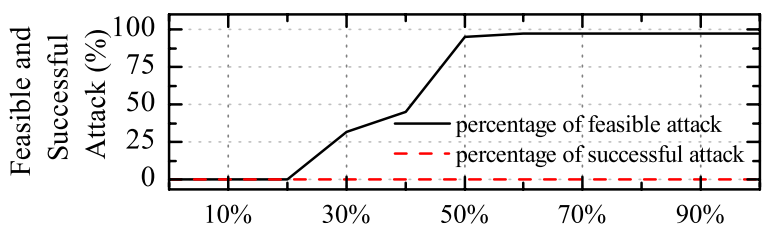

(c)

Load shift $\left(L_{S}\right)$ constraints

Figure 3. Statistic summary of 38 attack scenarios for the omnipotent attacker with the non-congested system.

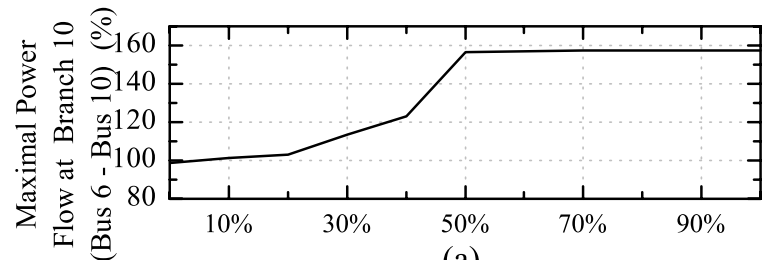

(a)

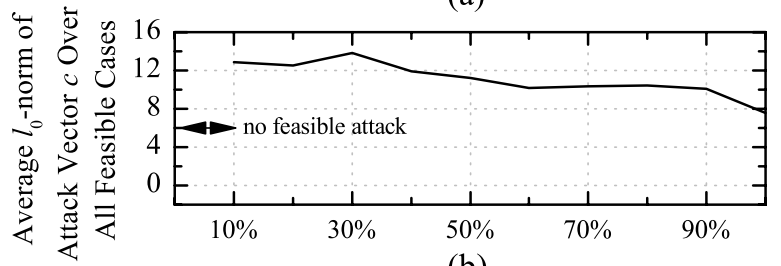

(b)

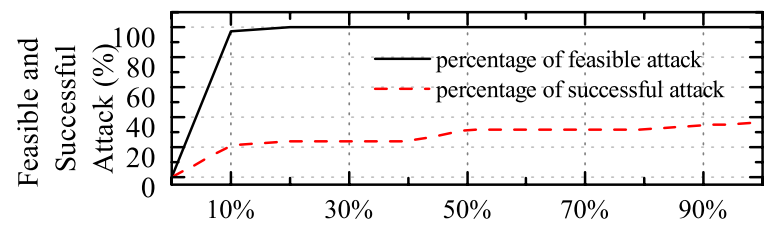

(c)

Load shift $\left(L_{S}\right)$ constraints

Figure 4. Statistic summary of 38 attack scenarios for the omnipotent attacker with the congested system.

subplots in both Figs. 3 and 4 Subplot (a) shows the maximal power flow on branch 10 (based on our observation, this is the attack with the maximal power flow, i.e., the worst-case attack); subplot (b) shows the average $l_{0}$-norm of attack vector $c$ over all feasible cases; and subplot (c) shows the percentage of feasible and successful attacks.

For both non-congested and congested scenarios, we ob- 
serve that the maximal power flow increases as $L_{S}$ constraint relaxes in Figs. 3(a) and 4(a). In Fig. 4(a), we observe a plateau after $L_{S}>50 \%$. It is due to the generator location and capacity limitation and the fact that the line flow on branch 10 cannot be increased anymore. From Figs. 3 (b) and 4 (b), as $L_{S}$ constraint relaxes, it is easier to attack the system since the average $l_{0}$-norm decreases and the attacker needs to change fewer bus states. It is due to the fact, for some cases, that the maximal power is saturated when the $L_{S}$ constraint relaxes. The attacker effectively concentrates the change of loads on fewer buses with heavy loads therefore changes fewer bus states. From Figs. 3(c) and 4(c), we observe that the attacker can find more feasible cases as $L_{S}$ constraint relaxes. Even if the attacker has full control over the system meters, its influence over the system is extremely limited by the load shift constraint. For instance, from Fig. 3(c), when $L_{S}=20 \%$, the attacker cannot find any feasible attacks while the attacker can find 12 feasible attacks when $L_{S}=30 \%$.

Comparing Figs. 3 and 4 the congested system is more vulnerable to our FDI attack. For a non-congested system, from Fig. 3 (c), the attacker cannot generate any successful attack. On the other hand, in Fig. 4(c), the feasible and successful attack percentage increases as $L_{S}$ constraint increases for the congested system. This is expected because the RTS-24bus system has redundant transmission capacity for reliability reasons and reducing all the line ratings proportionally will create a more stressed system. In conclusion, a congested system is naturally favored by the attacker. Thus, for the rest of the simulation, we only consider the congested system to illustrate the attack consequences.

Now we discuss the $l_{1}$-norm constraint. To understand the effect of the sparsity constraint, we fix the $L_{S}$ constraint and solve the proposed optimization problem for different $l_{1}$ norm constraint $\left(N_{1}\right)$ and for all target branches. In Fig. 5 . the maximal power flow on the target branch is plotted as a function of the $l_{1}$-norm constraint for a successful attack on target branch 17. The kink in Fig. 5 represents point of which the attack is large enough to cause a different set of generators to be dispatched.

Fig. 6 illustrates the effect of the $\gamma$ term in the objective function of our optimization problem for target branch 17. There are three sub-plots illustrating the following as a function of the $l_{1}$-norm constraint $N_{1}$ : (a) the maximal power flow, (b) the $l_{1}$-norm , and (c) the $l_{0}$-norm of the attack vector, respectively. In each subplot, we plot two curves, one with $\gamma$ set to zero and one with the chosen weight of $\gamma$ coefficient. Subplot (a) demonstrates that the $\gamma$ term does not decrease the resulting maximal power flow at all. Subplot (b) shows that once the maximal power flow saturates, introducing the $\gamma$ term causes the optimization problem to find the smallest attack vector in $l_{1}$-norm. This result in a stabilization of the $l_{0}$-norm as shown in subplot (c) in contrast to the $\gamma=0$ case.

\section{B. Attack consequences for a non-linear model}

We now use the attack vector from the optimization problem to perform the AC attack described in Sec. [III-C] If the attacker keeps injecting false data, the attack as well as the overload on

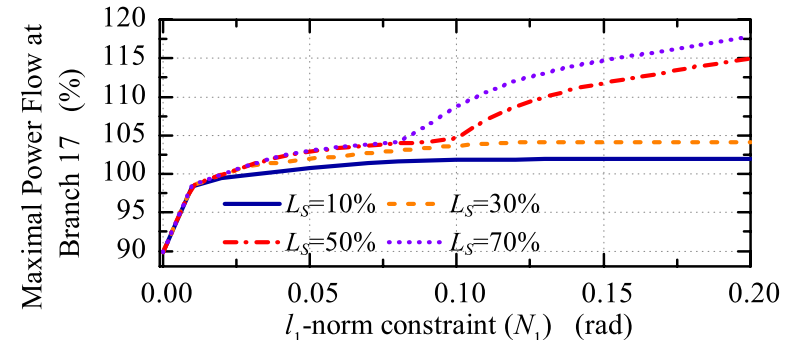

Figure 5. The maximal power flow v.s. the $l_{1}$-norm constraints with different load shift tolerance at target branch 17 (bus 10- bus 12).

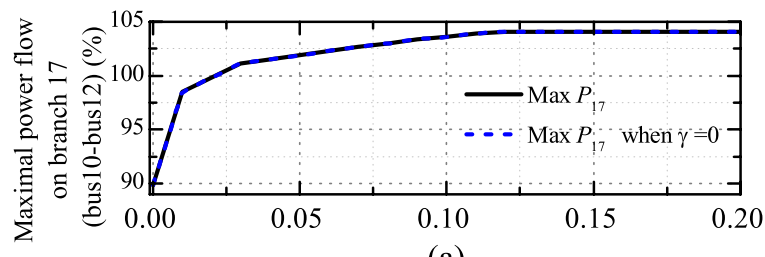

(a)

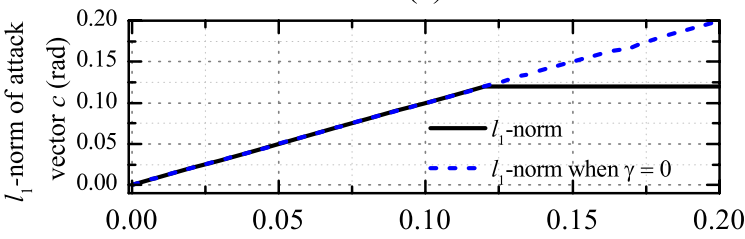

(b)

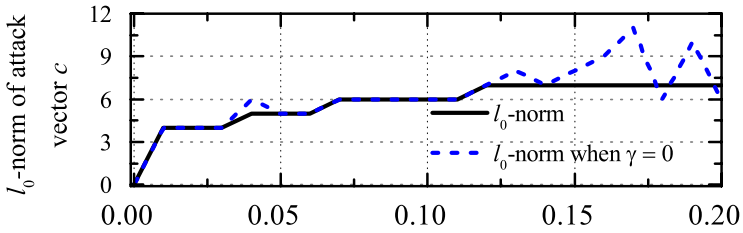

(c)

$l_{1}$-norm constraint $\left(N_{1}\right)$ with load shift $\left(L_{S}\right)=30 \%(\mathrm{rad})$

Figure 6. The $l_{1}$-norm and $l_{0}$-norm of solved attack vector $c$ v.s. the $l_{1}$ norm constraint $\left(N_{1}\right)$ when load shift $\left(L_{S}\right)$ is limited by $30 \%$; target branch 17 (bus $10-$ bus 12).

the branches will be sustained until the system configuration changes.

In this subsection, we assume a system with a complete set of measurements, i.e., both active and reactive power flows are measured at two ends of each branch and both active and reactive injection are measured at each load bus, which makes 186 measurements in total. Measurement error, as in (1), is assumed to have zero mean and variance $10^{-4}$. During the simulation, we assume the physical load is unchanged. Note that, to make the system congested, all ratings of the branches are decreased by $50 \%$. However, because of the existence of the reactive power, the convergence of ACOPF is not guaranteed. Thus, in order to compare AC and DC attack, certain ratings of branches in ACOPF have to be relaxed manually. We solve the optimization problem for target branch 17 and $L_{S}=30 \%$. Fig. 7 (a) compares the maximal power flow from the optimization problem (recall: DCOPF used in the optimization problem) with the physical power flow (active and apparent power) in the non-linear system after attack. In this scenario, the rating of branch 10 (bus 6-bus 


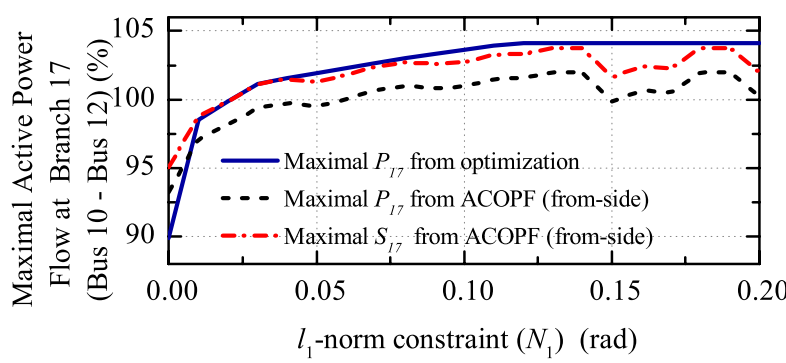

(a) Comparison of DC optimization solution and AC maximal active/apparent power flow on target branch 17

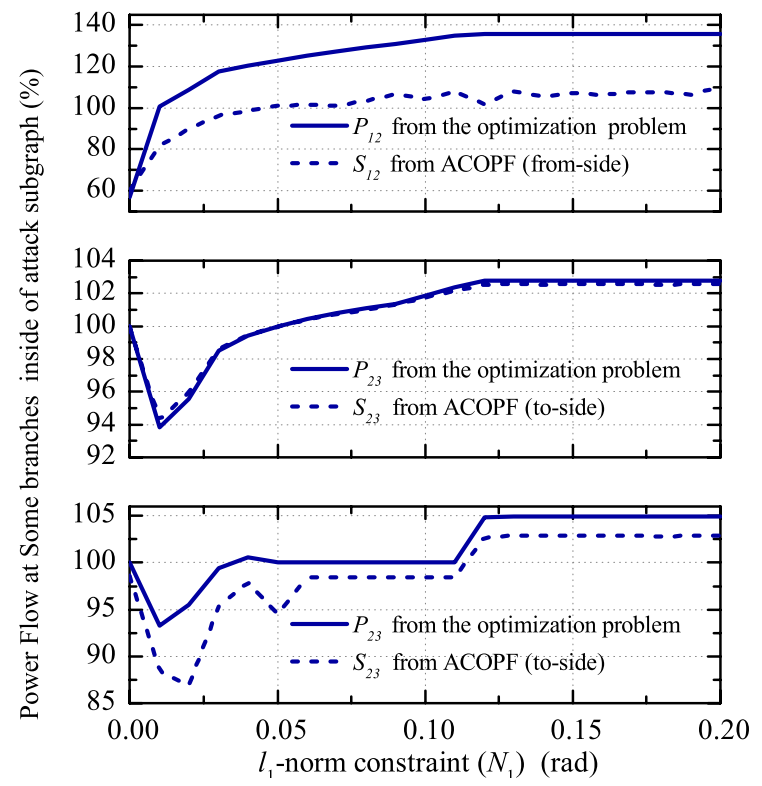

(b) Power flow on branch 12, 23, and 28

Figure 7. Attack simulation on AC system model

10) are relaxed to 145 MVA. Note that in the absent of the attack, i.e., $N_{1}=0$, the power flow for $\mathrm{AC}$ and DC OPFs result in sightly different power flow, however, as the attacker size is increased, the power flows closely track each other. In particularly, the AC attack successfully overloads branch 17 . Branches as 12, 23, and 28 are also overloaded even though the attacker has not targeted on them, as shown in Fig. 7 (b). Since branch 23 and 28 are congested prior to attack, once the generation is redispatched as a result of the attack, the power flow on these branches will change and in some cases it leads to overloads. Branch 12, while not congested prior to attack, suffers an overload due to the fact that it lies in the path of power delivering to branch 17.

\section{CONCLUSIONS AND FUTURE WORK}

This paper analyzed the physical consequences of false data injection attacks on power system state estimation. An attack framework was introduced in which the attacker matches the non-linear AC system characteristics by implementing local AC state estimation to a small number of measurements. Subsequently, a linear optimization problem was formulated to find the worst-case line overload attack. Numerical simulation was performed to test the resulting attacks on the IEEE-RTS24-bus system. We found that, aside from the size of the attack subgraph, the constraint that an attack not cause significant observed load shift at the control center significantly impacts the attacker's ability to overload a branch. Still, there exists attacks with mild load shift that cause overloads.

Extensions include attacks targeted to overload multiple lines; this was an inadvertent side effect of our attacks, but a more targeted effort may cause more extreme damage or even cascading outages. Secondly, the linear optimization problem may be extended to a more accurate non-linear problem. Finally, using accurate load statistics to detect abnormal load patterns caused by FDI attacks could further restrict the space of undetectable attacks.

\section{REFERENCES}

[1] Y. Liu, M. K. Reiter, and P. Ning, "False data injection attacks against state estimation in electric power grids," in in Proc. 16th ACM Conference on Computer and Communication Security, 2009, pp. 21-32.

[2] A. Teixeira, S. Amin, H. Sandberg, K. Johansson, and S. Sastry, "Cyber security analysis of state estimators in electric power systems," in 2010 49th IEEE Conference on Decision and Control (CDC), 2010, pp. 59915998.

[3] O. Kosut, L. Jia, R. J. Thomas, and L. Tong, "Malicious data attacks on smart grids," IEEE Transactions on Smart Grid, vol. 2, pp. 645-658, 2011.

[4] F. Pasqualetti, R. Carli, and F. Bullo, "A distributed method for state estimation and false data detection in power networks," in 2011 IEEE International Conference on Smart Grid Communications (SmartGridComm), 2011, pp. 469-474.

[5] H. Sandberg, A. Teixeira, and K. H. Johansson, "On security indices for state estimators in power networks," in Proc. 1st Workshop Secure Control Syst., 2010

[6] M. Rahman and H. Mohsenian-Rad, "False data injection attacks against nonlinear state estimation in smart power grids," in Power and Energy Society General Meeting (PES), 2013 IEEE, July 2013, pp. 1-5.

[7] J. Kim and L. Tong, "On topology attack of a smart grid: undetectable attacks and countermeasures," Selected Areas in Communications, IEEE Journal on, vol. 31, no. 7, pp. 1294-1305, July 2013.

[8] J. Kim, L. Tong, and R. Thomas, "Data framing attack on state estimation with unknown network parameters," in Signals, Systems and Computers, 2013 Asilomar Conference on, Nov 2013, pp. 1388-1392.

[9] D. Kundur, X. Feng, S. Liu, T. Zourntos, and K. Butler-Purry, "Towards a framework for cyber attack impact analysis of the electric smart grid," in Smart Grid Communications (SmartGridComm), 2010 First IEEE International Conference on, Oct 2010, pp. 244-249.

[10] L. Xie, Y. Mo, and B. Sinopoli, "Integrity data attacks in power market operations," Smart Grid, IEEE Transactions on, vol. 2, no. 4, pp. 659666, Dec 2011.

[11] A. Giani, R. Bent, M. Hinrichs, M. McQueen, and K. Poolla, "Metrics for assessment of smart grid data integrity attacks," in Power and Energy Society General Meeting, 2012 IEEE, July 2012, pp. 1-8.

[12] L. Jia, J. Kim, R. Thomas, and L. Tong, "Impact of data quality on real-time locational marginal price," Power Systems, IEEE Transactions on, vol. 29, no. 2, pp. 627-636, March 2014.

[13] G. Hug and J. Giampapa, "Vulnerability assessment of AC state estimation with respect to false data injection cyber-attacks," IEEE Transactions on Smart Grid, vol. 3, no. 3, pp. 1362-1370, 2012.

[14] J. Liang, O. Kosut, and L. Sankar, "Cyber attack on AC state estimation: Unobservability and physical consequences," in Power and Energy Society General Meeting, 2014 IEEE, July 2014.

[15] J. Salmeron, K. Wood, and R. Baldick, "Analysis of electric grid security under terrorist threat," Power Systems, IEEE Transactions on, vol. 19, no. 2, pp. 905-912, May 2004.

[16] Y. Yuan, Z. Li, and K. Ren, "Modeling load redistribution attacks in power systems," Smart Grid, IEEE Transactions on, vol. 2, no. 2, pp. 382-390, June 2011.

[17] —, "Quantitative analysis of load redistribution attacks in power systems," Parallel and Distributed Systems, IEEE Transactions on, vol. 23, no. 9, pp. 1731-1738, Sept 2012. 
[18] A. Abur and A. G. Expósito, Power system state estimation: theory and implementation. CRC, 2000.

[19] L. V. Stephen Boyd, Convex Optimization. Cambridge University Press, 2004.

[20] J. Fortuny-Amat and B. McCarl, "A representation and economic interpretation of a two-level programming problem," The Journal of the Operational Research Society, vol. 32, no. 9, pp. pp. 783-792, 1981. [Online]. Available: http://www.jstor.org/stable/2581394 